\title{
Giant Hemophilic Pseudotumor of the Iliopsoas: Case Report
}

\author{
Umit Y. MALKAN ${ }^{1}$, Gursel GUNES ${ }^{1}$, Eylem ELIACIK ${ }^{1}$, Ayse ISIK ${ }^{1}$, \\ Salih AKSU1, Bulent ATILLA², Yahya BUYUKASIK ${ }^{1}$ \\ ${ }^{1}$ Hacettepe University Faculty of Medicine Department of Hematology \\ ${ }^{2}$ Hacettepe University Faculty of Medicine Department of Orthopedics, Ankara, TURKEY
}

\begin{abstract}
Hemophilic pseudotumor results from multiple episodes of hemorrhage into bones or soft tissue spaces. It is a rare complication of severe hemophilia that occurs in $12 \%$ of the patients. Many cases have been published in the literature and different therapeutical approaches have been suggested. Here we describe a patient with giant hemophilic pseudotumor that had become incurable due to its size.
\end{abstract}

A 48-years-old male had been diagnosed with hemophilia A in 1966. Since 2005, he was followed up by Hacettepe Hematology Clinic as severe hemophilia with factor 8 levels $1 \%$. Approximately one and a half year ago, he started to feel pain in his left hip and in July 2013 he applied to our emergency service by bleeding mass on left hip. He did not take any factor 8 prophylaxis until July 2013. He was hospitalized and factor VIII replacement was started. Left hip was visualized by tomography (Figure 1). A 23x21x14 cm sized pseudotumor originating from left iliac area and expanding outside of pelvis by eroding the iliac bone and a left psoas muscle hematoma with $8.5 \mathrm{~cm}$ diameter were seen. The hematoma had internal septa. The bleeding was thought to be secondary to fistula of the pseudotumor to skin. On his retrospective evaluation, it was noticed that he had been diagnosed with iliopsoas pseudotumor 8 years ago in 2005. At that time he had been evaluated for an abdominal mass on physical examination in another center and a 97x93 mm liquefied hematoma located in the anterior part of the psoas muscle and posterior to this lesion a $74 \mathrm{x} 43 \mathrm{~mm}$ heterogeneously contrasted psoas muscle mass had been observed. No interventions had been planned. On follow-ups these masses had been re-evaluated in December 2010 and May 2011 by ultrasound. The diameters were relatively stable. $88 \times 88 \mathrm{~mm}$ and $75 \times 55$ $\mathrm{mm}$ in 2010 and $92 \times 77 \times 66$ and $78 \times 47$ in 2011, respectively. Iliac bone erosion had not been reported at these evaluations.

On his admission to our center in July 2013, the patient was evaluated by orthopedics, plastic surgery and interventional radiology clinics but surgery or intervention were not planned because of the size of the pseudotumor and operational risks. On August 2013 when the patient presented with fever a repeat computed tomography revealed air in the pseudotumor which was considered to indicate infection. Antibiotics were given and surgical drainage with VAC dressing was performed and nearly $1000 \mathrm{ml}$ hemorrhagic fluid was drained however no significant improvement observed in control tomography (Figure 2). Because the surgical treatment was out of choice radiotherapy was planned. In September 2013, 200 cGy/ day (2000 cGy in total) radiotherapy was applied on the mass. After all that interventions magnetic resonance imaging revealed no significant improvement: a pseudotumor covering left hemipelvis, gluteal region and retroperitoneum. No further surgery was planned and the patient was discharged by 1500 units of factor VIII, 3 days a week and VAC drainage. On 11 December 2013, the patient applied again to the emergency service with hip pain, fever and bleeding on gluteal region. 


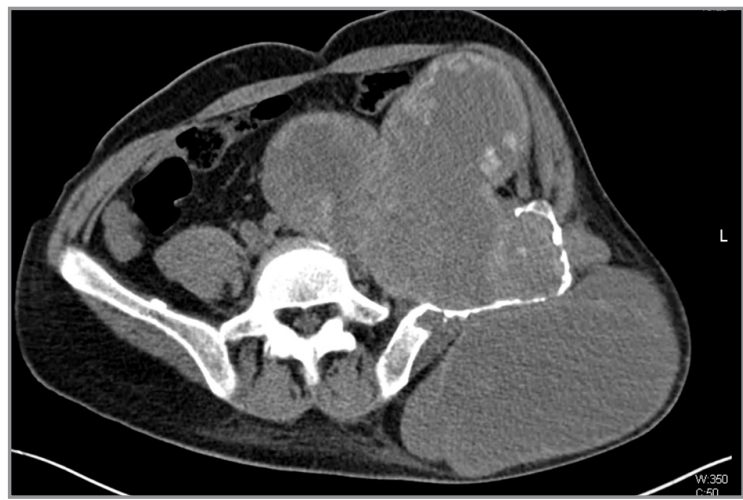

Figure 1. CT image of the patient before drainage and VAC treatment

Control tomography revealed that the hematoma and pseudotumor were stable and air was present in the pseudotumor. Due to huge size of the pseudotumor, the left kidney was replaced to anterior and superior position. Intestinal loops were also replaced to left. Main iliac artery and external iliac artery were compressed by the mass. The patient was evaluated by cardiovascular surgery department with these findings and again no surgical treatment was offered. Air in the pseudotumor was interpreted as recurrent infection and antibiotics were given. The patient is still on follow-up in the hematology clinic with prophylactic factor replacement with 1500 units 3 days per week since October 2013. He is still considered inoperable due to massive size of the pseudotumor.

The hemophilic pseudotumor is rare and serious complication of the typical hematomas. It manifests as a painless cystic mass of slow and irregular growth that may compress vital organs or open to skin by a fistula. ${ }^{1}$ The mechanism of pseudotumor of bone is not understood well. It was postulated that recurrent intraosseous hemorrhage into closed spaces prohibits blood reabsorption and promotes bone destruction thus showing its nature to continuously enlarge. ${ }^{2}$ Hemophilic pseudotumor has X-ray characteristics of both benign and malignant bone tumors, such as bone destruction, periosteal reaction, and new bone formation. ${ }^{3}$

In the patient in this case report, surgical treatment was considered to be too risky. Many inoperable cases that were managed by radiotherapy have been reported. ${ }^{2,4}$ The mechanism of the response of the hemophilic pseudotumor to radiation therapy is not yet known. Brant and Jordan suggested that 1000-

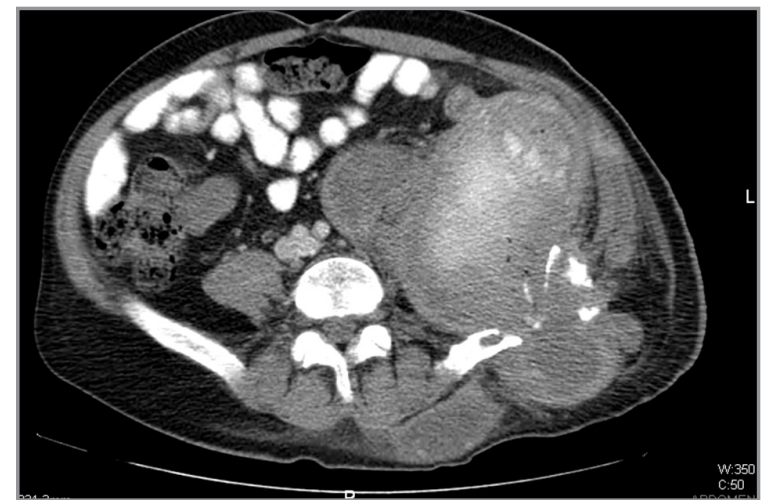

Figure 2. CT image of the patient after drainage and VAC treatment

$2000 \mathrm{cGy}$ radiotherapy leads to an endarteritis in an acutely bleeding hematoma. ${ }^{5}$ Reinhold suggested that the radiation may directly injure the fine vessels supplying the pseudotumor causing fibrosis, leading to eventual healing. ${ }^{6}$ However our case did not respond well to $2000 \mathrm{cGy}$ radiotherapy. Without any treatment hemophilic pseudotumors can become enormous in size and they may cause compression of nearby organs or they may present with bone destruction. At the same time they can lead to bleeding that affects the patient's life quality. Generally the hemophilic pseudotumors do not cause pain but very large lesions may cause pain due to compression of adjacent tissues.

In this case, the patient's iliac bone was still not eroded and the mass was relatively small in 2011. Maybe interventions or surgery could be successfully applied at that time. Or another option would be close periodical follow-ups. By this way, possibly it would be likely to diagnose earlier and halt pelvic erosion and skin fistulization by drainage, surgery and/or radiotherapy. In conclusion hemophilic pseudotumors, especially very large masses, can become inoperable, life threatening disease and interventional or surgical treatment methods should be performed before it is too late in a growing pseudotumor.

\section{REFERENCES}

1. Garcia-Perez R, Torres-Salmeron G., Sanchez-Bueno F., Garcia-Lopez A, Parrilla-Paricio P. Intraabdominal hemophilic pseudotumor: case report. Rev Esp Enferm Dig 100: $275-$ 280, 2008. 
2. Kang JO, Cho YJ, Yoo MC, Hong SE, Hemophilic pseudotumor of the ulna treated with low dose radiation theraphy: a case report. J Korean Med Sci 15: 601-603, 2000.

3. Liu B, Liu L, Feng Y, Li L. A Case Report on the Surgical Treatment of the Huge Inflammatory Pseudotumor in the AIDS Patient with Hemophilic. Case Rep Pathol 2011: 798649. doi: 10.1155/2011/798649, 2011.

4. Espandar R, Heidari P, Rodriguez-Merchan EC. Management of haemophilic pseudotumours with special emphasis on radiotherapy and arterial embolization. Haemophilia 15: 448457, 2009.

5. Brant EE, Jordan HH. Radiologic aspects of haemophilic pseudotumors in bone. Am J Roentgenol Radium Ther Nucl Med 115: 525-539, 1972.

6. Reinhold HS. The influence of radiation on blood vessels and circulation 4. Structural changes in blood vessels. Curr Top Radiat Res Q 10: 58-74, 1974.

\section{Correspondence}

Ümit Yavuz MALKAN

Mutlukent Mahallesi, Mutluköy Sitesi

1951. Sokak, No: 6

Ümitköy/ ANKARA-TURKEY

Mail: umitmalkan@hotmail.com

Tel: (+90.532) 7780087

Fax: $+90-3123051614$ 